\title{
Clinical Effect of a Topical Herbal Ointment on Pain in Temporomandibular Disorders: A Randomized Placebo-Controlled Trial
}

\author{
Larry C.F. Li, M.Orth., Ricky W.K. Wong, Ph.D., and A. Bakr M. Rabie, Ph.D.
}

\begin{abstract}
Objectives: The aim of this study was to compare the effectiveness of using Ping On ointment and using petroleum jelly in the treatment of temporomandibular joint (TMJ) and masticatory muscle pain, in order to establish the true efficacy of Ping On ointment.

Methods: In this randomized, double-blinded, placebo-controlled trial, 55 subjects with TMJ and/or masticatory pain (Group I patients according to the Research Diagnostic Criteria for Temporomandibular Disorder (RDC/ TMD) received Ping On ointment for 4 weeks, or placebo for 4 weeks. Subjects were evaluated with standard measures of efficacy: pain intensity measured by visual analogue scale and maximal comfortable mandibular opening, at baseline and again after 4 weeks of treatment.

Results: Ping On ointment significantly reduced the symptoms of painful TMJs and/or masticatory muscles. Maximal comfortable mandibular opening also improved in the Ping On ointment group compared with the placebo, but was not clinically significant.

Conclusions: This preliminary study suggests that topical application of Ping On ointment may be considered for further investigation as a potential first-line treatment modality, before prescribing analgesics, for the management of TMDs. It is topically applied, safe, reversible, and effective in managing TMDs and masticatory muscle pain.
\end{abstract}

\section{Introduction}

$\mathbf{T}$ EMPOROMANDIBULAR DISORDERS (TMDs) is a collective term that includes a number of clinical diagnoses involving the temporomandibular joints (TMJs), the masticatory musculature, and associated structures or both excluding pain of dental origin. ${ }^{1}$

TMDs are frequently encountered in general clinical practice, and their prevalence in the general population has been reported as being as high as $12 \%{ }^{2}$ Pain can be present any stage of TMDs and is a significant part of the symptoms that prompt patients to seek treatment. ${ }^{3}$

Treatments for TMDs are wide-ranging and directed primarily toward relief from persistent orofacial pain. Up to now, most TMDs have been treated by conservative treatments such as counseling, physiotherapy, splint therapy, and prescription of analgesics. Anti-inflammatory or analgesic medications - in particular, nonsteroidal anti-inflammatory drugs (NSAIDs) and nonselective cyclo-oxygenase inhibitorsare frequently used to manage TMDs. ${ }^{4-6}$ However, no treatments are considered completely effective and able to produce definite long-term pain relief. Therefore, patients with TMD pain usually report serious suffering and anxiety even when receiving treatment, which can last long periods and involve complicated procedures such as construction of occlusal splints, while not offering much promise. Given the prevalence of TMDs, safety concerns with chronic NSAID administration, and the controversial effectiveness of nonpharmacologic therapies such as TMJ surgery, splints, and occlusal alteration, new treatment modalities for TMDs are continually being sought.

Topical application of medicinal creams is one of the treatment alternatives drawing increasing attention. Topical application of capsaicin cream (Theraflex-TMJ cream, Sagmel Inc., IL), NSAIDs, and indomethacin phonophoresis have been shown to be effective in various studies. ${ }^{7-10}$

Chinese orthopedics is a field of Traditional Chinese Medicine (TCM) that has been used in the Chinese population for the treatment of bone, joint, and muscle diseases for several thousand years. One of the methods for treatment of musculoskeletal pain is local massage with topical Chinese medicinal herbs ointment, such as Ping On ointment. ${ }^{11}$ 
Ping On ointment (Ping On Ointment Company Limited, Hong Kong) is a licensed topical medicinal ointment of which the main ingredients are natural Chinese medicinal herbs. It has been registered in Hong Kong since 1965 and has been used extensively as a soothing massage balm for muscular aches, strain, and sprain. The main ingredients of Ping On ointment, according to the manufacturer, include peppermint oil, $18 \%$; menthol, $20 \%$; natural camphor, $6 \%$; birch oil, $6 \%$; sandalwood oil, $1 \%$; eucalyptus oil, $4 \%$; bee wax, $8 \%$; and aromatic oil, 3\%. It does not contain antibiotics, steroids, cortisone, or preservatives. If topical application of the ointment can be effective for decreasing the severity of TMD muscular pain, it could be endorsed as a new alternative for treating TMD muscular pain.

Our group published a case study of five consecutive cases using Ping On ointment to treat TMD muscular pain. ${ }^{11}$ All cases resulted in complete remission of pain within 1 month of topical massage. They concluded that this treatment method had high potential to benefit a significant number of people and that randomized controlled trials should be performed.

\section{Materials and Methods}

\section{Aim and objective}

The aim of the study is to compare the effectiveness of using Ping On ointment and using petroleum jelly in the treatment of TMJ and masticatory muscle pain (Group I patients according to the Research Diagnostic Criteria for Temporomandibular Disorder (RDC/TMD), ${ }^{12}$ in order to establish the true efficacy of Ping On ointment.

The null hypothesis states that there is no significant improvement in pain relief in patients receiving Ping On ointment compared with those receiving petroleum jelly.

The study was a randomized, double-blinded, placebocontrolled clinical trial. The CONSORT statement was used as guidance to ensure the quality of the study. ${ }^{13}$

\section{Study population}

Ethical approval was obtained from the Institutional Review Board of the University of Hong Kong/Hospital Authority Hong Kong West Cluster. The appropriate subjects were identified and recruited from specialist clinics and were also recruited from the public using newspaper advertising. In the latter case, all recruited subjects were referred to appropriate specialist departments for consultation if any doubt existed about the diagnosis or any pathology other than TMJ muscular pain suspected. The nature, aim, procedures, and possible risks and benefits of the study were explained to the eligible subjects. Both verbal and written informed consents were obtained prior to the screening. All of the subjects were referred to appropriate specialist departments for follow-up after the project upon their request.

\section{Inclusion criteria}

The inclusion criteria were the following.

1. Positive clinical diagnosis of Group I (muscle disorders) of TMDs. The TMD diagnosis is classified using axis I of the Research Diagnostic Criteria (RDC) for TMDs. ${ }^{12}$ Muscle disorders include both painful and nonpainful disorders. This classification deals only with the most common painful disorders associated with TMDs.
2. For joint and muscle pain complaint, subjects were required to self-report of at least 1 month of daily or nearly daily pain.

3. Subjects with myogenic pain were included if they met inclusion and exclusion criteria since patients with TMDs are known to exhibit muscle pain secondary to their joint dysfunction.

\section{Exclusion criteria}

The exclusion criteria were the following.

1. Subjects with infectious arthritis, crystal-induced arthropathies, musculoskeletal disorders, subjects with a primary diagnosis of myofascial pain based on the RDC.

2. Subjects with pain attributable to confirmed migraine or head pain condition other than tension headache.

3. Subjects with acute infection or other significant disease of teeth, ears, eyes, nose, or throats; subjects with untreated depressive disorder or not on stable antidepressant medication for more than 6 months.

4. Subjects with dental diseases that required ongoing treatment, which would confound the evaluation of orofacial pain.

5. Subjects who are not competent in giving consents.

6. Pregnant or lactating women.

7. Subjects with sensitivity to the ingredients of Ping On ointment were excluded.

Patients who met the inclusion criteria were informed about the study design and advised that they had equal opportunity of receiving experimental ointment or the placebo ointment without knowing until the trial had ended. They were informed that they could stop participating in the study at any time without any prejudice. A written informed consent was obtained by each patient who entered the trial.

Sixty-three (63) subjects were screened in total, and 55 patients met the inclusion criteria and were recruited. The screening consisted of medical history, questionnaire, and clinical examination as baseline record. The questionnaire and clinical examination followed the format of the RDC/TMD booklet. $^{12}$

\section{Study protocol}

The eligible subjects were randomized using blocked randomization into one of the two groups: treatment group (Ping On ointment) and placebo group (petroleum jelly). A minor trace $(0.5 \%)$ of Ping On ointment was mixed with the petroleum jelly to provide some medicinal smell to the placebo. The people involved in the randomization and in preparation and distribution of study articles were independent from the investigators, and both the investigators and subjects were blinded as to the treatment allocation. While there were differences in the texture, color, and odor of the placebo and active ointment, the investigators did not see either ointment at any time and were instructed not to ask any questions regarding the ointment used by a subject. The ointment to be given to participants was sealed in an opaque, tightly sealed container and then a bag in which no smell could be detected. The possibility of contamination was minimized by having the subjects attend their clinic visits separately and being asked not to bring the ointment to the clinic. The participants 
were also asked not to apply the ointment on the day of assessment.

During the study period, eligible subjects were advised to continue any other ongoing treatment such as medication or splint therapy for the condition at the same level as usual. They, however, were requested not to commence any other new intervention. Careful checks of the consumption of analgesics were made. In principle, there should be none, for any reason. The participants needed to report any medications taken or any treatment received during the experimental phase.

Subjects were asked to complete a diary at home daily, which included pain rating on a self-administrated $100-\mathrm{mm}$ Visual Analogue Scale (VAS) diary for 7 days before receiving the experimental or placebo ointment. Then, the Ping On ointment and petroleum jelly were distributed to the already allocated subjects by an independent person. The subjects were asked to complete the daily diary at home including the VAS, use of oral analgesics, and occurrence of adverse effects if there were any.

The participants were taught and given a demonstration on how to rub the ointment over the painful area and then to massage in a circular motion for 5 minutes twice a day. The area of application was just on the skin around the TMJ and affected muscles, which were usually the temporalis and masseters. Painful lateral and medial pterygoid muscles could not be approached due to anatomical inaccessibility. The subjects were reviewed every 2 weeks. After 4 weeks of ointment application, subjects received a final assessment in the clinic. Subjects were instructed to record whether there was any adverse effect daily since using the ointment and to report back to the investigator at the earliest convenience.

\section{Primary outcome}

The primary outcome was the efficacy in the treatment of TMJ and muscle pain as measured by a VAS. Subjects needed to complete the VAS once daily in the evening for 7 days as baseline diary of pain, and daily thereafter for 4 weeks of application of the ointment/control. The VAS consists of a 100-mm line, anchored with the extremes of pain intensity represented as "no pain" and "worst pain possible." VAS has been shown to provide a robust, sensitive, and reproducible method of measuring pain. ${ }^{14}$

\section{Secondary outcome}

The secondary outcome was to assess the mandibular function and the vertical mouth opening, which was the maximal comfortable mandibular opening measured in millimeters at the subject's maximum incisor-to-incisor mouth opening using a ruler. This measure of mandibular movement was defined as the maximal interincisal distance a subject can open without experiencing evoked pain. This measure has previously been shown to be valid and reliable., 15

\section{Statistical analysis}

The data were analyzed using SPSS standard statistical package (SPSS Statistical Software, Chicago, IL). Homogeneity of treatment for gender, age, and pain duration was analyzed by $\chi^{2}$ test. Pain diaries collected daily by VAS for 5 weeks were assessed by two-way analysis of variance and Tukey's multiple-comparison tests. Maximum mandibular comfortable opening was analyzed with independent samples $t$ tests. Values are reported as mean \pm 1 standard deviation. All tests were considered statistically significant at $p<0.05$.

The determination of sample size for this study was based on a postulated improvement treatment effect in $50 \%$ of the active treatment group compared to placebo group. ${ }^{16} \mathrm{~A}$ sample size of 22 per treatment group was calculated a priori as sufficient to detect this difference with $80 \%$ power with an $\alpha$ level of 0.05 .

\section{Results}

Sixty-three (63) subjects were invited for screening evaluation at the Orthodontic Clinic, Faculty of Dentistry, the University of Hong Kong. At the completion of the screening evaluation, 55 subjects were recruited and 45 of them completed the study. Of the 10 subjects who did not complete the study, 5 withdrew from the study due to time constraint and 5 failed to return for follow-up (see Fig. 1 outlining subject flowchart through the study).

\section{Subject characteristics}

No significant differences between the treatment group and the placebo group were detected with respect to age, gender, or pain duration (Table 1).

\section{Primary outcome measures: Efficacy in the pain relief measured by VAS}

Both Ping On ointment and placebo groups showed improvement in the mean VAS scores for pain intensity. However, only the Ping On ointment group results showed statistical significance $(p<0.001)$. The Ping On ointment group started to show significant symptomatic improvement after day 15, in comparison with the placebo group, which started about 1 week after applying the ointment. This might be due to the anti-inflammatory and analgesic effect of Ping On ointment

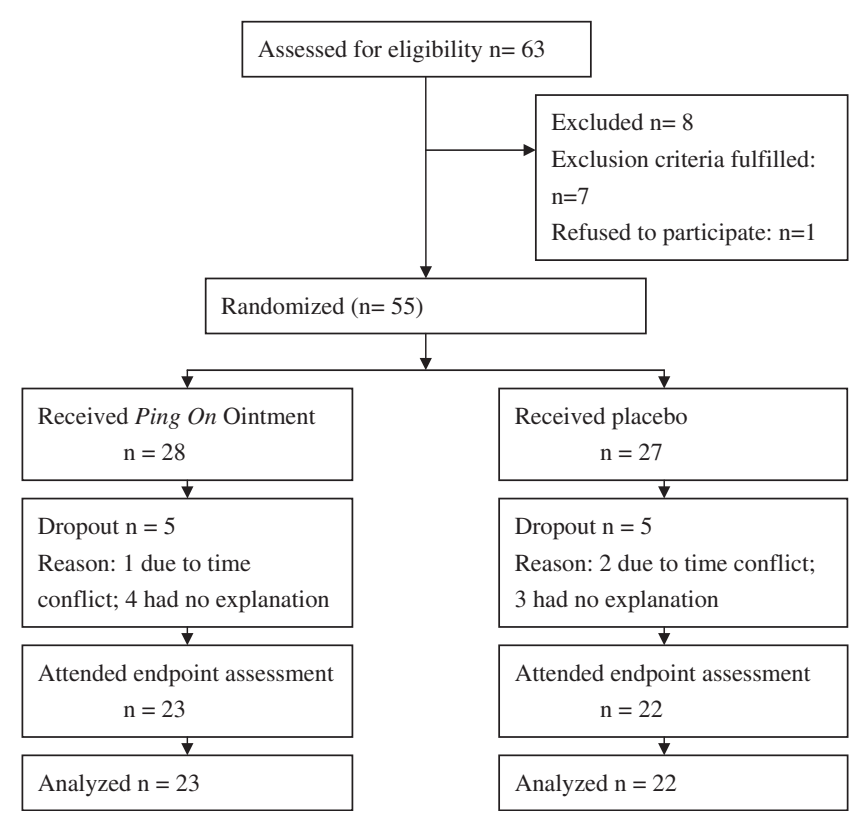

FIG. 1. Subject flowchart through the study. 
Table 1. Subject Baseline Characteristics $($ Mean \pm Standard Deviation [SD])

\begin{tabular}{lcc}
\hline & Ping On ointment & Placebo \\
\hline Sample size & 23 & 22 \\
Age (mean \pm SD) years & $43.96( \pm 13.127)$ & $47.14( \pm 9.296)$ \\
Female:male sex ratio & $17: 6$ & $15: 7$ \\
$\begin{array}{c}\text { Pain duration } \\
\text { (mean } \pm \text { SD) months }\end{array}$ & $29.61( \pm 31.442)$ & $35.82( \pm 31.526)$ \\
\hline
\end{tabular}

on the affected tissues. Two (2) of the subjects in the Ping On ointment group showed complete remission of pain at the final assessment.

Clinically significant pain reduction was defined as decrease of pain rating by $50 \%$ from the baseline pain rating during the treatment period. ${ }^{17}$ In the Ping On ointment group, the pain ratings reflected by VAS dropped 55\% from the baseline rating after completion of the treatment, which was considered clinically significant (Fig. 2, Table 2).

\section{Secondary outcome measures: Maximum comfortable mandibular opening measured in millimeters}

Subjects in both the Ping On ointment group and the placebo group had an increased maximum comfortable mandibular opening at the final visit as measured in millimeters compared to base records. However, only the Ping On ointment group showed a significant difference $(p<0.01)$ (Table 3$)$.

\section{Adverse effects}

All the reported adverse effects were mild in severity. There was no subject dropout due to adverse events. Eye irritation was among the most commonly reported. One (1) subject in the Ping On ointment group stopped the treatment for 3 days due to a burning sensation of the skin (Table 4).

\section{Discussion}

The results demonstrated that topical application of Ping On ointment was more effective than placebo in reducing pain from TMJ and masticatory muscles, and it reached a level of statistical significance. Therefore, the results support the hypothesis that there is significant improvement in pain relieving in patients receiving Ping On ointment compared with those receiving petroleum jelly. Consequently, the null hypothesis can be rejected.

The finding that improvement in pain rating in the Ping On ointment group was evident after 1 week of application (started from the 15th day including the first week when no ointment was applied), suggesting that the topical preparation has a rapid onset of action. The analgesic effect of Ping On ointment might be explained by the anti-inflammatory and anesthetic effect of its active ingredients. Study on the anti-inflammatory and analgesic effects of essential oils of eucalyptus showed that they possess neutrophil-dependent and independent anti-inflammatory activities as well as central and peripheral analgesic effects. ${ }^{18}$ Methyl salicylate (birch oil) is widely available in many over-the-counter analgesic preparations because of its anti-inflammatory and analgesic properties. ${ }^{19}$ Beeswax, commonly known as propolis, has proved successful in the treatment of chronic backache and hip pain, as well as fresh injuries of muscles and tendons. ${ }^{20}$ The ethanolic extract of propolis also has profound anti-inflammatory effects on both chronic and acute inflammation. ${ }^{21}$ Pain is at times related to ischemia due to tension or muscle spasm that can be improved by vaso-

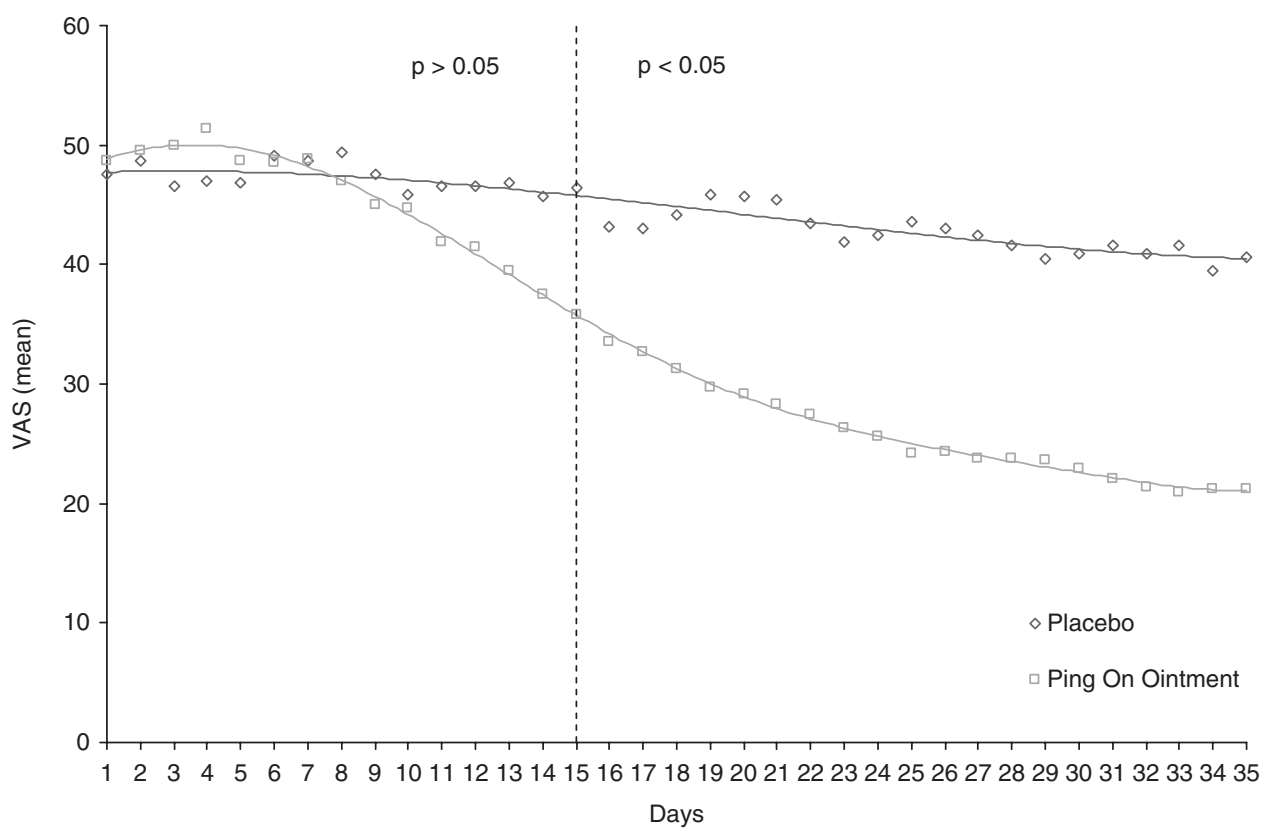

FIG. 2. Ping On ointment effect on temporomandibular joint and muscle pain intensity. Pain intensity daily time effect curve is shown as measured on a 100-mm visual analogue scale (VAS) for Ping On ointment and placebo. Data are expressed as mean values \pm standard error of the mean. Ping On ointment had significant analgesic effect on pain compared with placebo starting from day $15(p<0.05)$. Although there was a trend toward decrease in pain severity in the placebo group, there was no significant difference detected in this decrease in pain reduction compared to the baseline score. 
Table 2. Primary Outcome Measure with Pain INTENSITY

\begin{tabular}{lcclll}
\hline & \multicolumn{2}{c}{ Ping On ointment $(\mathrm{n}=23)$} & & \multicolumn{2}{c}{ Placebo $(\mathrm{n}=22)$} \\
\cline { 2 - 3 } \cline { 6 - 7 } & Mean & SD & & Mean & $S D$ \\
\hline Baseline & $49.35^{\mathrm{a}}$ & 14.44 & & $47.76^{\mathrm{a}}$ & 11.88 \\
Week 1 & $42.48^{\mathrm{a}}$ & 14.52 & & $46.92^{\mathrm{a}}$ & 13.14 \\
Week 2 & $31.50^{\mathrm{b}, \mathrm{c}}$ & 16.30 & & $44.81^{\mathrm{a}} \mathrm{b}$ & 12.43 \\
Week 3 & $25.09^{\mathrm{c}}$ & 17.34 & & $42.63^{\mathrm{a}, \mathrm{b}}$ & 12.84 \\
Week 4 & $21.93^{\mathrm{c}}$ & 18.08 & & $40.81^{\mathrm{a}, \mathrm{b}}$ & 14.44 \\
\hline
\end{tabular}

Visual analogue scale results were analyzed using two-way analysis of variance and Tukey's multiple-comparison tests.

a,b,c Groups identified by different superscripts were significantly different at $p<0.05$.

Two-way analysis of variance revealed that significant differences $(p<0.001)$ were observed for the factor "treatment" $(p<0.001)$ and factor "time" $(p<0.001)$. The interactions between these two factors were significant $(p<0.001)$.

$\mathrm{SD}$, standard deviation.

dilation, which brings more oxygen to the affected area. This anti-inflammatory and vasodilative nature of these ingredients might play a major role in the pain relief.

The speed of onset of pain relief observed in this study was an unexpected finding. While one can only speculate about the mechanisms underlying the clinical observations, it is possible that the active ingredients such as peppermint oil, menthol, camphor, and aromatic oil provided immediate analgesic activity, and the repeated application of these agents contributed to the observed results. Peppermint oil has been shown to be effective for relaxing and smoothing muscle and was effective in the treatment of tension and headache, and may have action on the vascular tissue. ${ }^{22}$ From previous studies, $10 \%$ peppermint oil in ethanol solution had been shown to alleviate tension-type headache. ${ }^{23}$ Peppermint has a high menthol content, which has a ubiquitous use in dermatology for its topical anesthetic and analgesic effects. ${ }^{24}$ Menthol has been used since antiquity for medicinal purposes, but it is only the relatively recent discovery of thermosensitive cation channels in the sensory nerves that finally provides the answer to how menthol can elicit the same cool sensation as low temperatures. ${ }^{24}$ From previous studies, it was found that menthol caused analgesia

Table 3. Ping ON Ointment and Placebo Effects on Maximum Comfortable Mandibular Opening FolloWing 4 WEEKS of TREATMENT

Mean maximum comfortable mandibular opening ( $\mathrm{mm}$ )

\begin{tabular}{lcc}
\cline { 2 - 3 } Week & $\begin{array}{c}\text { Ping On ointment } \\
(\mathrm{n}=23)\end{array}$ & $\begin{array}{c}\text { Placebo } \\
(\mathrm{n}=22)\end{array}$ \\
\hline Baseline & $31.48 \pm 11.457$ & $33.18 \pm 12.385$ \\
Week 4 & $35.91 \pm 11.453$ & $34.00 \pm 12.067$ \\
Mean difference & $-4.435 \pm 6.680$ & $-0.818 \pm 3.404$ \\
$\begin{array}{l}\text { Significance } \\
\text { (2-tailed) }\end{array}$ & $0.004^{\mathrm{a}}$ & 0.272 \\
\hline
\end{tabular}

Values are mean \pm standard deviation.

a Ping On ointment group $(4.435 \mathrm{~mm})$ had significant improvement with greater change in mandibular movement $(p<0.01)$ compared to placebo group $(0.818 \mathrm{~mm})$.
Table 4. Incidence of Adverse Events Reported DuRING THE 4-WeEk Study

\begin{tabular}{lcc}
\hline $\begin{array}{l}\text { Reported adverse } \\
\text { events }\end{array}$ & $\begin{array}{c}\text { Ping On ointment } \\
(\mathrm{n}=23)\end{array}$ & $\begin{array}{c}\text { Placebo } \\
(\mathrm{n}=22)\end{array}$ \\
\hline Eye irritation & 6 & 0 \\
Burning sensation & 3 & 0 \\
Itchiness & 1 & 1 \\
\hline
\end{tabular}

by desensitizing nociceptive $C$ fibers ${ }^{25}$ and/or by its activation of TRPM8 and/or inhibition of TRPA1. ${ }^{26}$ Galeotti et al. ${ }^{27}$ demonstrated that menthol has a strong dose-dependent local anesthetic action. In topical use, menthol has been shown to facilitate the penetration of other drugs through the epidermis. ${ }^{28}$ Its ability as a penetration enhancer makes it an ideal vehicle for topical formulation. ${ }^{24}$ Camphor is a naturally occurring compound that is used as a major active ingredient of balms and liniments supplied as topical analgesics. Despite its long history of common medical use, the underlying molecular mechanism of camphor action is not understood. Capsaicin and menthol, two other topically applied agents widely used for similar purposes, are known to excite and desensitize sensory nerves by acting on two members of transient receptor potential channel superfamily. Camphor has been shown to activate them. ${ }^{29}$ Aromatic oil was also shown to have analgesic effect in the treatment of lower back pain. ${ }^{30}$ Local massage increases the local circulation to increase the transmission of the drug.

Traditional Chinese Medicine recognizes TMDs as a type of arthromyodynia (Bi syndrome). ${ }^{31}$ Arthromyodynia is mainly manifested as body pain, swelling, stiffness, and tendon spasm. $^{32}$ The most common type is the anemofrigid-damp arthralgia, which is caused by the Wind-Cold Damp pathogen. Arthralgia, in the theory of TCM, has the meaning of blockage and obstruction. The blockage of the meridians, vessels, bones, muscles, tendons, and skin result in the pain. Thus, the treatment principle is to activate the medians and collaterals, promote the circulation, and relieve the stagnation. The mechanism is therefore to relieve Wind, concurrently disperse Cold, and eliminate Dampness. ${ }^{33}$ According to the properties of Chinese medicinal herbs, peppermint ${ }^{34}$ and eucalyptus ${ }^{35}$ have the action of expelling the Wind, and sandalwood $^{35}$ and camphor ${ }^{35}$ can dry the Dampness and clear the Cold. Bee wax $^{35}$ and sandalwood ${ }^{35}$ promote the circulation of medians and collaterals, while peppermint ${ }^{34}$ and camphor $^{35}$ disperse stagnation.

Movement-evoked pain in mouth opening is a common measure in TMDs and muscular pain and also had been used in recent studies of TMD treatments since this measure directly reflects the patient's jaw function as well as improvements in signs and symptoms. ${ }^{2,36}$ For painful TMD conditions, a minimum of 9-mm increase in mandibular range of motion is considered to be a clinically significant improvement following a therapeutic intervention. ${ }^{37,38}$ In our finding, subjects in the Ping On ointment group had significantly greater change $(4.435 \mathrm{~mm})$ in the mandibular maximal comfortable opening relative to the placebo group $(0.818 \mathrm{~mm})$. However, this has not achieved the $9-\mathrm{mm}$ standard to be considered as clinically significant. This might be due to the limited penetration effect of the topical medicinal ingredients of the ointment to the tissue and also the limited duration of application. 
Nevertheless, the improved mandibular mobility observed seemed correlated with those seen in daily reported pain intensity at rest. This finding indicated that Ping On ointment might have effect on reducing both evoked pain and pain at rest or spontaneous pain.

In this 4-week study, Ping On ointment showed safety and high tolerability at the recommended doses. No major adverse event was observed or reported. However, eye irritation and mild burning sensation of the skin seemed to be common complications of using Ping On ointment. The high incidence of eye irritation could be explained by the proximity of the TMJ area to the eyes. The patients should be advised to avoid applying the ointment too close the eyes. Particular attention should be paid when going to sleep, as it may increase the risk of having the ointment enter the eyes.

In this study, the subjects were screened by clinical examinations and self-reported questionnaires. Ideally, the diagnosis should be made with a combination of magnetic resonance imaging or computed tomography.

This study reported the short-term effect of Ping On ointment. The results only applied to the immediate effect of using Ping On ointment for up to 4 weeks. The sample size of the present study was rather small. A multicenter study stratifying subjects into those with predominantly muscle pain and those with joint pain in a long-term follow-up period would give more conclusive results and information regarding the long-term benefit of this topical ointment.

Further questions that need to be asked are: What actually caused the therapeutic effect? Was it the properties of the ointment or the regular effects of the massage when the ointment was being applied? How do we know that the ointment did not just provide an effective lubricant for massage like that shown for other forms of manual therapy? Does it have a neurophysiologic effect on descending pain inhibitory systems? All of these are important considerations in future clinical trials testing the ointment's effectiveness. Further research is also needed to investigate the effect of the Ping On ointment on the joint tissue at the biochemical and molecular levels and to identify the combined effects of different active ingredients involved.

Nonetheless, the results of this study showed that topical application of Ping On ointment may be considered for further investigation as a potential first-line treatment modality, before prescribing analgesics, in the management of TMDs. It is topically applied, safe, reversible, and effective in managing TMDs and masticatory muscle pain.

\section{Acknowledgments}

The authors particularly want to thank Mr. Shadow Yeung for the statistical analysis. This research was supported by a grant from The University of Hong Kong.

ClinicalTrial.gov Identifier: NCT00812604.

\section{Disclosure Statement}

No competing financial interests exist.

\section{References}

1. National Institutes of Health Technology Assessment Conference on Management of Temporomandibular Disorders.
Bethesda, Maryland, April 29-May 1, 1996. Proceedings. Oral Surg Oral Med Oral Pathol Oral Radiol Endod 1997;83:49-50.

2. Ta LE, Dionne RA. Treatment of painful temporomandibular joints with a cyclooxygenase- 2 inhibitor: A randomized placebo-controlled comparison of celecoxib to naproxen. Pain 2004;111:13-21.

3. Rugh JD, Solberg WK. Oral health status in the United States: Temporomandibular disorders. J Dent Educ 1985;49:398406.

4. Antczak-Bouckoms A. Reaction paper to chapter 12 and 13. In: Sessle BJ, Bryant PS, Dionne RA, eds. Temporomandibular disorders and related pain conditions, Seattle, WA: IASP Press, 1995:237-245.

5. Dionne RA. Pharmacologic treatments for temporomandibular disorders. Oral Surg Oral Med Oral Pathol Oral Radiol Endod 1997;83:134-142.

6. List T, Axelsson S, Leijon G. Pharmacologic interventions in the treatment of temporomandibular disorders, atypical facial pain, and burning mouth syndrome: A qualitative systematic review. J Orofac Pain 2003;17:301-310.

7. Winocur E, Gavish A, Halachmi M, et al. Topical application of capsaicin for the treatment of localized pain in the temporomandibular joint area. J Orofac Pain 2000;14:31-36.

8. Lobo SL, Mehta N, Forgione AG, et al. Use of Theraflex-TMJ topical cream for the treatment of temporomandibular joint and muscle pain. J Craniomandib Pract 2004;22:137-144.

9. Svensson P, Houe L, Arendt-Nielsen L. Effect of systemic versus topical nonsteroidal anti-inflammatory drugs on postexercise jaw-muscle soreness: A placebo-controlled study. J Orofac Pain 1997;11:353-362.

10. Shin SM, Choi JK. Effect of indomethacin phonophoresis on the relief of temporomandibular joint pain. Cranio 1997; 15:345-348.

11. Wong RWK, Rabie ABM. Local message with topical analgesic, a novel treatment modality for temporomandibular muscular pain, a case study report of 5 consecutive cases. Open Orthop J 2008;2:97-102.

12. Dworkin SF, LeResche L. Research diagnostic criteria for temporomandibular disorders: Review, criteria, examinations and specifications, critique. J Craniomandib Disord 1992;6: 301-355.

13. Moher D, Schulz KF, Altman DG. The CONSORT statement: Revised recommendations for improving the quality of reports of parallel-group randomised trials. Lancet 2001;357: 1191-1194.

14. Cohen M, Wolfe R, Mai T, Lewis D. A randomized, double blind, placebo controlled trial of a topical cream containing glucosamine sulfate, chondroitin sulfate, and camphor for osteoarthritis of the knee. J Rheumatol 2003;30:523-528.

15. Wright EF, Schiffman EL. Treatment alternatives for patients with masticatory myofascial pain. J Am Dent Assoc 1995; 126:1030-1039.

16. Simon LS, Lanza FL, Lipsky PE, et al. Preliminary study of the safety and efficacy of SC-58635, a novel cyclooxygenase 2 inhibitor: Efficacy and safety in two placebo-controlled trials in osteoarthritis and rheumatoid arthritis, and studies of gastrointestinal and platelet effects. Arthritis Rheum 1998;41: 1591-1602.

17. Turk DC, Rudy TE, Sorkin BA. Neglected topics in chronic pain treatment outcome studies: Determination of success. Pain 1993;57:253-254.

18. Silva J, Abebe W, Sousa SM, et al. Analgesic and antiinflammatory effects of essential oils of eucalyptus. J Ethnopharmacol 2003;89:277-283. 
19. Chan TY. Potential dangers from topical preparations containing methyl salicylate. Hum Exp Toxicol 1996;15:747-750.

20. Golder W. Propolis. The bee glue as presented by the GraecoRoman literature. Wurzbg Medizinhist Mitt 2004;23:133-145.

21. Park EH, Kahng JH. Suppressive effects of propolis in rat adjuvant arthritis. Arch Pharm Res 1999;22:554-558.

22. Kligler B, Chaudhary S. Peppermint oil. Am Fam Physician 2007;75:1027-1030.

23. Göbel H, Fresenius J, Heinze A. Effectiveness of Oleum menthae piperitae and paracetamol in therapy of headache of the tension type. Nervenarzt 1996;67:672-681.

24. Patel T, Ishiuji Y, Yosipovitch G. Menthol: A refreshing look at this ancient compound. J Am Acad Dermatol 2007;57:873-878.

25. Cliff MA, Green BG. Sensory irritation and coolness produced by menthol: Evidence for selective desensitization of irritation. Physiol Behav 1994;56:1021-1029.

26. Macpherson LJ, Dubin AE, Evans MJ, et al. Noxious compounds activate TRPA1 ion channels through covalent modification of cysteines. Nature 2007;445:541-545.

27. Galeotti N, Di Cesare, Mannelli L, et al. Menthol: A natural analgesic compound. Neurosci Lett 2002;322:145-148.

28. Yener G, Gönüllü $U$, Uner $M$, et al. Effect of vehicles and penetration enhancers on the in vitro percutaneous absorption of celecoxib through human skin. Pharmazie 2003;58: 330-333.

29. Xu H, Blair NT, Clapham DE. Camphor activates and strongly desensitizes the transient receptor potential vanilloid subtype 1 channel in a vanilloid-independent mechanism. J Neurosci 2005;25:8924-8937.

30. Dyszkiewicz A, Opara J. Monitoring the treatment of low back pain using non-steroid anti-inflammatory drugs and aromatic oil components. Ortop Traumatol Rehabil 2006; $8: 210-218$

31. Lin HB. Using Traditional Chinese Medicine for treating temporomandibular disorders [in Chinese]. Chin J Tradit West Med 2003;6.
32. Ye YY, Jiang Y, Ma XQ. Professor Zhu Liangchun's experience in differentiating and treating $B i$-syndromes. Shanghai J Tradit Chin Med 2003;9.

33. Ke N-m, Ke H, Wang X-y. Treatment of BI syndrome with TCM method and compared the clinical effect and utilization of commonly used governs the Bi syndrome Chinese herb medicine. Chin Arch Tradit Chin Med 2007;1.

34. Zuo YF, Tang DC, Xuan JY, et al. Science of Chinese materia medica. Shanghai, PRC: Publishing house of Shanghai University of Traditional Chinese Medicine, 2003:57.

35. Nan Jing Chinese Medicine University, Traditional Chinese Medicine Dictionary [in Chinese]. Shanghai: Publishing House of Shanghai Science and Technology, 2006.

36. Smith P, Mosscrop D, Davies S, et al. The efficacy of acupuncture in the treatment of temporomandibular joint myofascial pain: A randomised controlled trial. J Dent 2007;35: 259-267.

37. Kropmans TJ, Dijkstra PU, Stegenga B, et al. Smallest detectable difference in outcome variables related to painful restriction of the temporomandibular joint. J Dent Res 1999; 78:784-789.

38. Kropmans T, Dijkstra P, Stegenga B, et al. Smallest detectable difference of maximal mouth opening in patients with painfully restricted temporomandibular joint function. Eur J Oral Sci 2000;108:9-13.

Address correspondence to: Ricky W.K. Wong, Ph.D. Orthodontics Department University of Hong Kong, 2/F Prince Philip Dental Hospital 34 Hospital Road Sai Ying Pun Hong Kong

E-mail: fyoung@hkucc.hku.hk 
